\title{
A new approach for solving systems of fractional differential equations via natural transform
}

\author{
A. S. Abedl-Rady ${ }^{1}$, S. Z. Rida ${ }^{1}$, A. A. M. Arafa ${ }^{2}$, and H. R. Abedl-Rahim ${ }^{1}$ \\ ${ }^{1}$ Department of Mathematics, Faculty of Science, South Valley University, Qena, Egypt. \\ ahmed1safwat1@hotmail.com, \\ szagloul@yahoo.com, \\ hamdy.ragab2013@yahoo.com , \\ ${ }^{2}$ Department of Mathematics and Computer Science, Faculty of Science Port Said University, Port Said, \\ anaszi2@yahoo.com.
}

\begin{abstract}
In this paper, A new method proposed and coined by the authors as the natural variational iteration transform method(NVITM) is utilized to solve linear and nonlinear systems of fractional differential equations. The new method is a combination of natural transform method and variational iteration method. The solutions of our modeled systems are calculated in the form of convergent power series with easily computable components. The numerical results shows that the approach is easy to implement and accurate when applied to various linear and nonlinear systems of fractional differential equations.
\end{abstract}

Keywords: Natural transform, Natural variational iteration transform method (NVITM), Systems of fractional differential equations.

\section{Introduction}

The natural transform, initially was defined by Khan and Khan [1] as N - transform, who studied their properties and applications. Later , Belgacem et al. [2,3] defined its inverse and studied some additional fundamental properties of this integral transform and named it the Natural transform. Applications of Natural transform in the solution of differential and integral equations and for the distribution and Bohemians spaces can be found in $[3,4,5,6,7,8,9,10]$. Now,we mention the following basic definitions of natural transform.

\subsection{Natural Transform}

over the set of functions

$$
A=\left\{f(t): \exists M, \tau_{1}, \tau_{2}>0,|f(t)|<M e^{|t| / \tau_{j}}, \text { if } t \in(-1)^{j} \times[0, \infty)\right\}
$$

the natural transform $[1,15,16]$ of $f(t)$ is defined by

$$
N[f(t)]=R(s ; u)=\int_{0}^{\infty} f(u t) e^{-s t} d t, u>0, s>0
$$

where $N[f(t)]$ is the natural transformation of the time function $f(t)$ and the variables $u$ and $s$ are the natural transform variables.

\subsection{Natural - Laplace, and Sumudu Duality}

If $R(s, u)$ is Natural transform and $F(s)$ is Laplace transform of function $f(t)$ in A then, $G(u)$ is Sumudu transform of function $f(t)$ in $\mathrm{A}$, then [17]:

Natural- Laplace Duality (NLD) is

$$
N[f(t)]=R(s ; u)=\frac{1}{u} \int_{0}^{\infty} f(t) e^{-\frac{s t}{u}} d t=\frac{1}{u} F\left(\frac{s}{u}\right),
$$

Natural-Sumudu Duality (NSD) is

$$
N[f(t)]=R(s ; u)=\frac{1}{s} \int_{0}^{\infty} f\left(\frac{u t}{s}\right) e^{-t} d t=\frac{1}{s} G\left(\frac{u}{s}\right)
$$

\subsection{Natural transform of nth derivative}

If $f^{n}(t)$ is the nth derivative of function $f(t)$ then, its natural transform is given by[17]:

$$
N\left[f^{n}(t)\right]=R_{n}(s, u)=\frac{s^{n}}{u^{n}} R(s, u)-\sum_{k=0}^{n-1} \frac{u^{n-(k+1)}}{s^{n-k}} f^{(k)}(0), n \geq 1
$$

\subsection{Convolution theorem of Natural transform}

If $F(s, u), G(s, u)$ are the natural transform of respective functions $f(t), g(t)$ both defined in set A then[17],

$$
N\left[f^{*} g\right]=u F(s, u) G(s, u)
$$

where $f * g$ is convolution of two functions $f$ and $g$.

\subsection{Natural transform of fractional derivative}


If $N[f(t)]$ is the natural transform of the function $f(t)$, then the natural transform of fractional derivative of order $\alpha$ is defined as [17] :

$$
N\left[f^{(\alpha)}(t)\right]=\frac{s^{\alpha}}{u^{\alpha}} R(s, u)-\sum_{k=0}^{n-1} \frac{u^{\alpha-(k+1)}}{s^{\alpha-k}} f^{(k)}(0)
$$

\subsection{Weight shift property}

Let the function $f(t)$ belongs to set $\mathrm{A}$ be multiplied with weight funtion $e^{ \pm t}$ then [16],

$$
N\left[e^{ \pm t} f(t)\right]=\frac{s}{s \mp u} R\left[\frac{s u}{s \mp u}\right]
$$

\subsection{Change of scale property}

Let the function $f(a t)$ belongs to set $\mathrm{A}$, where a is non zero constant then [16],

$$
N[f(a t)]=\frac{1}{a} R\left[\frac{s}{a}, u\right]
$$

\section{Analysis of method}

In the case of an algebraic equation $f(x)=0$, the Lagrange multipliers can be evaluated by an iteration formula for finding the solution of the algebraic Equation $f(x)=0$ that can be constructed as[18];

$$
x_{n+1}=x_{n}+\lambda f\left(x_{n}\right) \text {. }
$$

The optimality condition for the extreme $\frac{\partial x_{n+1}}{\partial x_{n}}=0$ Leads to

$$
\lambda=-\frac{1}{f^{\prime}\left(x_{n}\right)}
$$

Where $\partial$ is the classical variational operator. From (9) and (10), for a given initial value $X_{0}$, we can find the approximate solution $X_{n+1}$ by the iterative scheme for (9) as follows:

$$
x_{n+1}=x_{n}-\frac{f\left(x_{n}\right)}{f^{\prime}\left(x_{n}\right)}, f\left(x_{0}\right) \neq 0, n=0,1,2, \ldots . .
$$

This algorithm is well known as the Newton-Raphson method and has quadratic convergence. To illustrate the basic idea of the natural variational iteration transform method, we consider the following fractional differential equation:

$$
{ }_{0}^{c} D_{t}^{\alpha} U+R[U(t)]+F[U(t)]=K(t), 0<\alpha
$$

Where $R$ is a linear operator, $\mathrm{F}$ is a nonlinear operator and $K(t)$ is a given continuous function. Now, we extend this idea to finding the unknown Lagrange multiplier. The main step is to first take the natural transform to Eq. (12). Then the linear part is transformed into an algebraic equation as follows:

$$
\frac{s^{\alpha}}{u^{\alpha}} R(s, u)-\frac{s^{\alpha} U(0)}{u^{\alpha}} \ldots \ldots \ldots . . .-\frac{s^{\alpha-(n)} U^{(n-1)}(0)}{u^{\alpha-(n-1)}}+N\left[R\left[U_{n}(t)\right]+F\left[U_{n}(t)\right]-K(t)\right]=0,
$$

Where $N[f(t)]=R(s ; u)=\int_{0}^{\infty} f(u t) e^{-s t} d t, u>0, s>0$

The iteration formula of (11) can be used to suggest the main iterative scheme involving the Lagrange multiplier as:

$$
R_{n+1}=R_{n}+\lambda\left(\frac{s^{\alpha}}{u^{\alpha}} R_{n}(s, u)-\frac{s^{\alpha} U(0)}{u^{\alpha}} \ldots \ldots \ldots . . . . .-\frac{s^{\alpha-(n)} U^{(n-1)}(0)}{u^{\alpha-(n-1)}}+N\left[R\left[U_{n}(t)\right]+F\left[U_{n}(t)\right]-K(t)\right]\right) .
$$

Considering $N\left[R\left[U_{n}(t)\right]+N\left[U_{n}(t)\right]\right.$ as restricted terms, one can derive a Lagrange multiplier as:

$\partial R_{n+1}=\partial R_{n}+\partial\left(\lambda \frac{s^{\alpha}}{u^{\alpha}} R_{n}\right)$

$\partial R_{n+1}=\partial R_{n}+\frac{s^{\alpha}}{u^{\alpha}}\left(\lambda^{\prime} R_{n}+\lambda \partial R_{n}\right)$

This yields the stationary conditions of Eq. (14) as follows;

$\left(1+\frac{s^{\alpha} \lambda}{u^{\alpha}}\right)=0$, 
$\frac{s^{\alpha}}{u^{\alpha}}\left(\lambda^{\prime} R_{n}\right)=0$

With Eq. (14) and the inverse-natural transform $N^{-1}$, the iteration formula (14) can be explicitly given as:

$$
\begin{aligned}
& U_{n+1}=U_{n}-N^{-1}\left[\frac{u^{\alpha}}{s^{\alpha}}\left(\frac{s^{\alpha}}{u^{\alpha}} R_{n}(s, u)-\frac{s^{\alpha} U(0)}{u^{\alpha}} \ldots \ldots \ldots . . .-\frac{s^{\alpha-(n)} U^{(n-1)}(0)}{u^{\alpha-(n-1)}}+N\left[R\left[U_{n}(t)\right]+F\left[U_{n}(t)\right]-K(t)\right]\right)\right] \\
& U_{n+1}=N^{-1}\left[\frac{u^{\alpha}}{s^{\alpha}}\left(\frac{s^{\alpha}}{u^{\alpha}} U(0) \ldots \ldots \ldots . . .-\frac{s^{\alpha-(n)} U^{(n-1)}(0)}{u^{\alpha-(n-1)}}\right)-\frac{u^{\alpha}}{s^{\alpha}}\left(N\left[R\left[U_{n}(t)\right]+F\left[U_{n}(t)\right]-K(t)\right)\right]\right. \\
& U_{0}(t)=N^{-1}\left[\frac{u^{\alpha}}{s^{\alpha}}\left(\frac{s^{\alpha}}{u^{\alpha}} U(0) \ldots \ldots \ldots . .-\frac{s^{\alpha-(n)} U^{(n-1)}(0)}{u^{\alpha-(n-1)}}\right)\right]
\end{aligned}
$$

Consequently the exact solution may be procured by using

\section{Applications}

$$
U(x, t)=\operatorname{Lim}_{n \rightarrow \infty} U_{n}(x, t)
$$

In this section, we illustrate the applicability, simplicity, and efficiency of NVITM for solving systems of linear and nonlinear fractional differential equations.

\subsection{Application 1}

Consider the following system of linear FPDEs[19]:

$$
\begin{array}{ll}
D_{t}^{\alpha} U=V_{x}-U-V, & t>0,0<\alpha \leq 1, x \in R \\
D_{t}^{\alpha} V=U_{x}-U-V &
\end{array}
$$

with the initial condition:

$$
U(x, 0)=\sinh x, V(x, 0)=\cosh x
$$

Applying the natural transform on both sides of Eq.(16) and Eq.(17), then

$$
\begin{aligned}
& N\left[D_{t}^{\alpha} U\right]=N\left[V_{x}-U-V\right], \\
& N\left[D_{t}^{\alpha} V\right]=N\left[U_{x}-U-V\right]
\end{aligned}
$$

Using the properties of natural transform we obtain;

$$
\begin{aligned}
& \frac{s^{\alpha}}{u^{\alpha}} R_{n}(s, u)-\frac{s^{\alpha-1}}{u^{\alpha}} U(x, 0)-N\left[V_{x}-U-V\right]=0 \\
& \frac{s^{\alpha}}{u^{\alpha}} R_{n} *(s, u)-\frac{s^{\alpha-1}}{u^{\alpha}} V(x, 0)-N\left[U_{x}-U-V\right]=0
\end{aligned}
$$

The iteration formula of Eq.(16) and Eq.(17)can be constructed as:

$R_{n+1}=R_{n}+\lambda(s, u)\left[\frac{s^{\alpha}}{u^{\alpha}} R_{n}(s, u)-\frac{s^{\alpha-1}}{u^{\alpha}} U(x, 0)-N\left[V_{n x}-U_{n}-V_{n}\right]\right]$

$R^{*}{ }_{n+1}=R *_{n}+\lambda *(s, u)\left[\frac{s^{\alpha}}{u^{\alpha}} R_{n} *(s, u)-\frac{s^{\alpha-1}}{u^{\alpha}} V(x, 0)-N\left[U_{n x}-U_{n}-V_{n}\right]\right]$

where $\lambda, \lambda *$ is a general Lagrange multiplier, which can be identified optimally via the variational theory, $\tilde{U}_{0}=0$, $\left.N\left[V_{n x}-U_{n}-V_{n}\right]\right]$ and $\left.N\left[U_{n x}-U_{n}-V_{n}\right]\right]$ are a restricted variation, that are, $\partial \tilde{U}_{n}=0$

i.e.

$$
\begin{array}{ll}
\partial R_{n+1}=\partial R_{n}+\partial\left(\lambda \frac{s^{\alpha}}{u^{\alpha}} R_{n}\right), & \partial R_{n+1}=\partial R_{n}+\frac{s^{\alpha}}{u^{\alpha}}\left(\lambda^{\prime} R_{n}+\lambda \partial R_{n}\right) \\
\partial R^{*}{ }_{n+1}=\partial R *_{n}+\partial\left(\lambda * \frac{s^{\alpha}}{u^{\alpha}} R *_{n}\right) & \partial R^{*}{ }_{n+1}=\partial R *_{n}+\frac{s^{\alpha}}{u^{\alpha}}\left(\lambda^{\prime} * R{ }_{n}+\lambda * \partial R *_{n}\right)
\end{array}
$$

This yields the stationary conditions, which gives $\lambda=-\frac{u^{\alpha}}{s^{\alpha}}, \lambda^{*}=-\frac{u^{\alpha}}{s^{\alpha}}$.

Substituting this value of Lagrangian multiplier in Eq.(20) and Eq.(21), we get the following iteration formula:

$$
R_{n+1}=R_{n}-\frac{u^{\alpha}}{s^{\alpha}}\left[\frac{s^{\alpha}}{u^{\alpha}} R_{n}(s, u)-\frac{s^{\alpha-1}}{u^{\alpha}} U(x, 0)-N\left[V_{n x}-U_{n}-V_{n}\right]\right]
$$




$$
R^{*}{ }_{n+1}=R *_{n}-\frac{u^{\alpha}}{s^{\alpha}}\left[\frac{s^{\alpha}}{u^{\alpha}} R_{n} *(s, u)-\frac{s^{\alpha-1}}{u^{\alpha}} V(x, 0)-N\left[U_{n x}-U_{n}-V_{n}\right]\right]
$$

Applying inverse natural transform on both sides of Eq.(22) and Eq.(23),we get:

$$
U_{n+1}=\sinh x+N^{-1}\left[\frac{u^{\alpha}}{s^{\alpha}}\left[N\left[V_{n x}-U_{n}-V_{n}\right]\right]\right.
$$

$V_{n+1}=\cosh x+N^{-1}\left[\frac{u^{\alpha}}{s^{\alpha}}\left[N\left[U_{n n}-U_{n}-V_{n}\right]\right]\right.$,

$U(x, 0)=\sinh x, V(x, 0)=\cosh x$,

$U_{1}=\sinh x-\cosh x \frac{t^{\alpha}}{\Gamma(\alpha+1)}$,

$$
V_{1}=\cosh x-\sinh \frac{t^{\alpha}}{\Gamma(\alpha+1)}
$$

$U_{2}=\sinh x-\cosh x \frac{t^{\alpha}}{\Gamma(\alpha+1)}+\sinh x \frac{t^{2 \alpha}}{\Gamma(2 \alpha+1)}$,

$V_{2}=\cosh x-\sinh x \frac{t^{\alpha}}{\Gamma(\alpha+1)}+\cosh x \frac{t^{2 \alpha}}{\Gamma(2 \alpha+1)}$,

$U_{3}=\sinh x-\frac{t^{\alpha}}{\Gamma(\alpha+1)} \cosh x+\sinh x \frac{t^{2 \alpha}}{\Gamma(2 \alpha+1)}-\cosh x \frac{t^{3 \alpha}}{\Gamma(3 \alpha+1)}$,

$V_{3}=\cosh x-\sinh x \frac{t^{\alpha}}{\Gamma(\alpha+1)}+\cosh x \frac{t^{2 \alpha}}{\Gamma(2 \alpha+1)}-\sinh x \frac{t^{3 \alpha}}{\Gamma(3 \alpha+1)}$.

Finally, we approximate the analytical solution $U(x, t)$ and $V(x, t)$ by:

$U(x, t)=\operatorname{Lim}_{n \rightarrow \infty} U_{n}(x, t), \quad V(x, t)=\operatorname{Lim}_{n \rightarrow \infty} V_{n}(x, t)$,

$U(x, t)=\sinh x-\frac{t^{\alpha}}{\Gamma(\alpha+1)} \cosh x+\sinh x \frac{t^{2 \alpha}}{\Gamma(2 \alpha+1)}-\cosh x \frac{t^{3 \alpha}}{\Gamma(3 \alpha+1)}+\ldots$,

$V(x, t)=\cosh x-\sinh x \frac{t^{\alpha}}{\Gamma(\alpha+1)}+\cosh x \frac{t^{2 \alpha}}{\Gamma(2 \alpha+1)}-\sinh x \frac{t^{3 \alpha}}{\Gamma(3 \alpha+1)}+\ldots$,

$$
\begin{aligned}
& U(x, t)=\sinh x E_{2 \alpha, 1}\left(t^{2 \alpha}\right)-t^{\alpha} E_{2 \alpha, \alpha+1}\left(t^{2 \alpha}\right) \cosh x, \\
& V(x, t)=\cosh x E_{2 \alpha, 1}\left(t^{2 \alpha}\right)-t^{\alpha} E_{2 \alpha, \alpha+1}\left(t^{2 \alpha}\right) \sinh x
\end{aligned}
$$

Where $\sum_{k=0}^{\infty} \frac{\left(t^{\alpha}\right)^{k}}{\Gamma(\alpha k+1)}=E_{\alpha, 1}\left(t^{\alpha}\right)$ is the famous Mittag-Leffler function.

For the special case $\alpha=1$, we obtain (See Figures $(1,2)$ )

$U(x, t)=\sinh x E_{2,1}\left(t^{2}\right)-t^{\alpha} E_{2,2}\left(t^{2}\right) \cosh x$

$V(x, t)=\cosh x E_{2,1}\left(t^{2}\right)-t E_{2,2}\left(t^{2}\right) \sinh x$

Where $\quad E_{2,1}\left(t^{2}\right)=\cosh (t), \quad t E_{2,2}\left(t^{2}\right)=\sinh (t)$,

$U(x, t)=\sinh x \cosh t-\sinh t \cosh x=\sinh (x-t)$,

$V(x, t)=\cosh x \cosh t-\sinh t \sinh x=\cosh (x-t)$.

Hence; 


$$
\begin{gathered}
U(x, t)=\sinh (x-t) \\
V(x, t)=\cosh (x-t)
\end{gathered}
$$

Which is the exact solution and is same as obtained by [19].

\subsection{Application 2}

Consider the following system of time-fractional coupled Burgers equations[20]:

$$
\begin{aligned}
& D_{t}^{\alpha} U-U_{x x}-2 U U_{x}+(U V)_{x}=0, t>0,0<\alpha \leq 1, x \in R, \\
& D_{t}^{\alpha} V-V_{x x}-2 V V_{x}+(U V)_{x}=0,
\end{aligned}
$$

with the initial condition:

$$
U(x, 0)=\sin x \text { and } V(x, 0)=\sin x
$$

where $\alpha$ is parameter describing the order of the fractional derivative. The functions $U(x, t), V(x, t)$, are the unknown functions, $t$ is the time and $x$ is the spatial coordinate. The derivative is understood in the Caputo sense. The general response expression contains parameter describing the order of the fractional derivative that can be varied to obtain various responses.

By applying the natural transform on both sides of Eq.(38) and Eq.(39) ,then

$N\left[D_{t}^{\alpha} U\right]-N\left[U_{n x x}+2 U_{n} U_{n x}-\left(U_{n} V_{n}\right)_{x}\right]=0$

$N\left[D_{t}^{\alpha} V\right]-N\left[V_{n x x}+2 V_{n} V_{n x}-\left(U_{n} V_{n}\right)_{x}\right]=0$,

Using the properties of natural transform we obtain;

$\frac{s^{\alpha}}{u^{\alpha}} R_{n}(s, u)-\frac{s^{\alpha-1}}{u^{\alpha}} U(x, 0)-N\left[U_{n x}+2 U U_{x}-(U V)_{x}\right]=0$

$\frac{s^{\alpha}}{u^{\alpha}} R_{n} *(s, u)-\frac{s^{\alpha-1}}{u^{\alpha}} V(x, 0)-N\left[V_{x x}+2 V V_{x}-(U V)_{x}\right]=0$

The iteration formula of Eq.(38) and Eq.(39) can be constructed as:

$R_{n+1}=R_{n}+\lambda(s, u)\left[\frac{s^{\alpha}}{u^{\alpha}} R_{n}(s, u)-\frac{s^{\alpha-1}}{u^{\alpha}} U(x, 0)-N\left[U_{n x x}+2 U_{n} U_{n x}-\left(U_{n} V_{n}\right)_{x}\right]\right]$

$R^{*}{ }_{n+1}=R^{*}{ }_{n}+\lambda *(s, u)\left[\frac{s^{\alpha}}{u^{\alpha}} R(s, u)-\frac{s^{\alpha-1}}{u^{\alpha}} V(x, 0)-N\left[V_{n x x}+2 V V_{x x}-\left(U_{n} V_{n}\right)_{x}\right]\right]$

where $\lambda, \lambda *$ is a general Lagrange multiplier, which can be identified optimally via the variational theory, $\tilde{U}_{0}=0$

,$\left.N\left[U_{x n x}+2 U_{n} U_{n x}-\left(U_{n} V_{n}\right)_{x}\right]\right]$ and $\left.N\left[V_{n x x}+2 V_{n} V_{x x}-\left(U_{n} V_{n}\right)_{x}\right]\right]$ are a restricted variation, that are, $\partial \tilde{U}_{n}=0$ i.e.

$$
\begin{aligned}
& \partial R_{n+1}=\partial R_{n}+\partial\left(\lambda \frac{s^{\alpha}}{u^{\alpha}} R_{n}\right), \quad \partial R_{n+1}=\partial R_{n}+\frac{s^{\alpha}}{u^{\alpha}}\left(\lambda^{\prime} R_{n}+\lambda \partial R_{n}\right) \\
& \partial R^{*}{ }_{n+1}=\partial R^{*}{ }_{n}+\partial\left(\lambda * \frac{s^{\alpha}}{u^{\alpha}} R^{*}{ }_{n}\right) \quad, \quad \partial R^{*}{ }_{n+1}=\partial R^{*}{ }_{n}+\frac{s^{\alpha}}{v^{\alpha}}\left(\lambda^{\prime} * R^{*}{ }_{n}+\lambda_{1} \partial R^{*}{ }_{n}\right)
\end{aligned}
$$

This yields the stationary conditions, which gives $\lambda=-\frac{u^{\alpha}}{s^{\alpha}}, \lambda^{*}=-\frac{u^{\alpha}}{s^{\alpha}}$.

Substituting this value of Lagrangian multiplier in Eq.(43) and Eq.(44)we get the following iteration formula:

$$
\begin{aligned}
& R_{n+1}=R_{n}-\frac{u^{\alpha}}{s^{\alpha}}\left[\frac{s^{\alpha}}{u^{\alpha}} R_{n}(s, u)-\frac{s^{\alpha-1}}{u^{\alpha}} U(x, 0)-N\left[U_{n x x}+2 U_{n} U_{n x}-\left(U_{n} V_{n}\right)_{x}\right]\right] \\
& R^{*}{ }_{n+1}=R^{*}{ }_{n}-\frac{u^{\alpha}}{s^{\alpha}}\left[\frac{s^{\alpha}}{u^{\alpha}} R^{*}{ }_{n}(s, u)-\frac{s^{\alpha-1}}{u^{\alpha}} V(x, 0)-N\left[V_{n x x}+2 V_{n} V_{n x}-\left(U_{n} V_{n}\right)_{x}\right]\right]
\end{aligned}
$$

Applying inverse natural transform on both sides of Eq.(45) and Eq.(46)we get:

$$
\begin{aligned}
& U_{n+1}=U_{n}-N^{-1}\left[\frac{u^{\alpha}}{s^{\alpha}}\left[\frac{s^{\alpha}}{u^{\alpha}} R_{n}(s, u)-\frac{s^{\alpha-1}}{u^{\alpha}} U(x, 0)-N\left[U_{n x x}+2 U_{n} U_{n x}-\left(U_{n} V_{n}\right)_{x}\right]\right]\right], \\
& V_{n+1}=V_{n}-N^{-1}\left[\frac{u^{\alpha}}{s^{\alpha}}\left[\frac{s^{\alpha}}{u^{\alpha}} R_{n}(s, u)-\frac{s^{\alpha-1}}{u^{\alpha}} V(x, 0)-N\left[V_{x x}+2 V_{n} V_{x x}-\left(U_{n} V_{n}\right)_{x}\right]\right]\right], \\
& U_{0}=\sin x, \quad V_{0}=\sin x,
\end{aligned}
$$




$$
\begin{aligned}
U_{1} & =\sin x-\frac{t^{\alpha}}{\Gamma(\alpha+1)} \sin x, \\
V_{1} & =\sin x-\frac{t^{\alpha}}{\Gamma(\alpha+1)} \sin x, \\
U_{2} & =\sin x-\frac{t^{\alpha}}{\Gamma(\alpha+1)} \sin x+\frac{t^{2 \alpha}}{\Gamma(2 \alpha+1)} \sin x, \\
V_{2} & =\sin x-\frac{t^{\alpha}}{\Gamma(\alpha+1)} \sin x+\frac{t^{2 \alpha}}{\Gamma(2 \alpha+1)} \sin x .
\end{aligned}
$$

Finally, we approximate the analytical solution $U(x, t)$ and $V(x, t)$ by:

$$
U(x, t)=\operatorname{Lim}_{n \rightarrow \infty} U_{n}(x, t), \quad V(x, t)=\operatorname{Lim}_{n \rightarrow \infty} V_{n}(x, t)
$$

Hence,

$$
U(x, t)=V(x, t)=\sin x E_{\alpha, 1}\left(-t^{\alpha}\right)
$$

Where $\sum_{k=0}^{\infty} \frac{\left(-t^{\alpha}\right)^{k}}{\Gamma(\alpha k+1)}=E_{\alpha}\left(-t^{\alpha}\right) \quad$ is the famous Mittag-Leffler function.

For the special case $\alpha=1$, we obtain[See Figures $(3,4$,$) ]$

$$
U(x, t)=e^{-t} \sin x, \quad V(x, t)=e^{-t} \sin x
$$

which is the exact solution of obtained by [20] .

\section{Conclusion}

In this paper, a novel approach was introduced and utilized to solve linear and nonlinear systems of fractional differential equations. In this research work, it was emonstrated through different examples how the new method can be used for solving various systems of fractional differential equations. When compared with the existing published methods, it is easy to notice that the new method has many advantages. It is straightforward, easy to understand, and fast, requiring much less computations to perform a limited number of steps of the simple procedure that can be applied to find the exact solution of a wide range of types of systems of fractional differential equations. Furthermore, there is no need for using linearization or restrictive assumptions when employing this new

\section{Reference}

1. Waqar,A., Zafar, H., and Khan. 2008, N- transform - properties and applications, NUST J. Engg. Sci, 127-133.

2. Belgacem, F. B. M. and Silambarasan, R. 2012, Theory of natural transform, Math. Engg, Sci. Aerospace (MESA),99124 .

3. Belgacem, F. B. M. and Silambarasan, R. 2011,Applications of the natural transform to Maxwell's equations, Prog. Electromagnetic Research Symposium Proc. Suzhou, China, 899 - 902 .

4. Al-Omari, S. K. Q. 2013, On the applications of natural transform, International Journal of Pure and Applied Mathematics, $729-744$.

5. Bulut, H., Baskonus, H.M. and Belgacem, F. B. M. 2013, The analytical solution of some fractional ordinary differential equations by the Sumudu transform method, Abstract and Applied Analysis 2013. Article ID 2013875

6. Loonker, Deshna and Banerji, P. K. 2013,Natural transform for distribution and Boehmian spaces, Math. Engg. Sci. Aerospace, 69 - 76 .

7. Loonker, Deshna and Banerji, P. K. 2013,Natural transform and solution of integral equations for distribution spaces, Amer. J. Math. Sci.

8. Loonker, Deshna and Banerji, P. K. 2013, Applications of natural transform to differential equations, J. Indian Acad. Math, $151-158$.

9. Podlubny, I. 1999, Fractional differential equations, Mathematics in Science and Engineering, Academic Press, San Diego, USA. 
10. Mittag-Leffler, G.M. 1903,Sur la nouvelle function $\mathrm{E}_{\alpha}(\mathrm{x})$, C.R. Acad. Sci., Paris (Ser.II) , 554-558.

11. Mainardi ,F., Rionero and Ruggeeri, T. 1994, On the initial value problem for the fractional diffusion-wave equation, In waves and stability in continuous media, World Scientific, Singapore,246-251.

12. Chaurasia. V. B. L., and Singh, J. 2010,Application of Sumudu transform in Schrödinger equation occurring in quantum mechanics, Applied Mathematical Sciences,2843-2850.

13. Jumarie.2009, via the Mittag-Leffler function and modified order Riemann-Liouville derivative,Applied Mathematics Letters, 1659-1664.

14. Jumarie.2009, Table of some basic fractional calculus formulae derived from a modified Riemann- Liouvillie derivative for non differentiable functions, Applied Mathematics Letters, 378-385

15. Belgacem , F. B. M. and Silambarasan, R. 2012, Advances in the natural transform. American Institute of Physics, AIPConference Proceeding, 106-110.

16. Belgacem, F. B. M. and Silambarasan, R. 2012, Theory of natural transform. Mathematics In Engineering, Science and Aerospace (MESA), 99-124.

17. Deshna, Loonker and Banerji1, P. K. 2013, Solution of fractional ordinary differential equations by natural transform, International Journal of Mathematical Engineering and Science , 2277-6982.

18. Wu, G.C. and Baleanu, D. 2013, Variational iteration method for fractional calculus - a universal approach by Laplace transform.

19. Karbalaie, A. , Muhammed, H. H. and Erlandsson, B.E. 2013 ,Using Homo-Separation of Variables for Solving Systems of Nonlinear Fractional Partial Differential Equations, International Journal of Mathematics and Mathematical Sciences, 1-8.

20. Liu, J. , Hou, G. 2011, Numerical solutions of the space- and time-fractional coupled Burgers equations by generalized differential transform method, Applied Mathematics and Computation ,27001-7008.

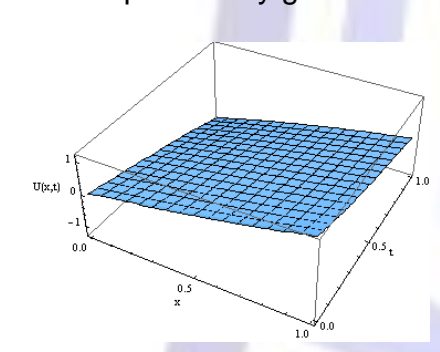

(a)

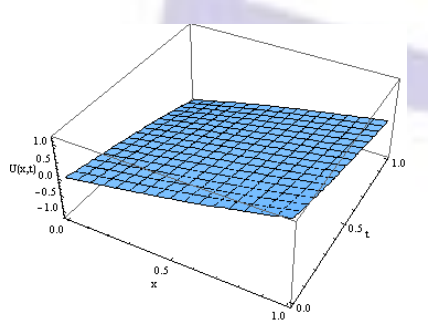

(c)

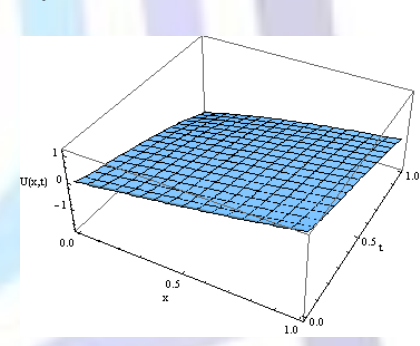

(b )

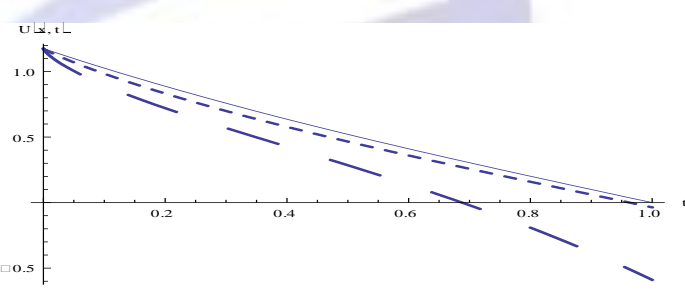

(d)

Figure 1:The surface plot of the solution $U(x, t)$ or $\mathrm{V}(\mathrm{x}, \mathrm{t})$ of application 1 when (a) $\alpha=0.75$, (b) $\alpha=0.90$, (c) $\alpha=1$ which is the exact solution and Plots of $U(x, t)$ or $\mathrm{V}(\mathrm{x}, \mathrm{t})$ versus $t$ at $x=1$ for different values of

$(--) \alpha=0.75,(-) \alpha=0.90$, ( - ) $\alpha=1$ which is the exact solution. 


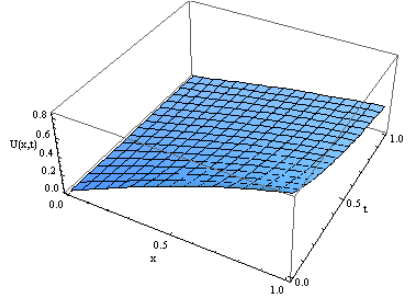

(a)

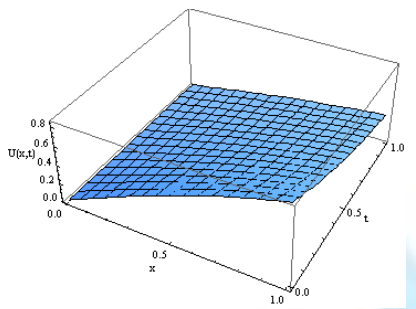

(c)
Journal of Advances in Mathematics

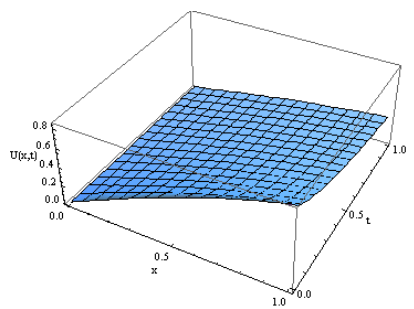

(b)

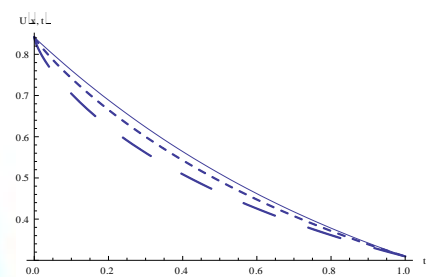

(d)

Figure 2:The surface plot of the solution $U(x, t)$ or $\mathrm{V}(\mathrm{x}, \mathrm{t})$ of application 2 when (a) $\alpha=0.75$, (b) $\alpha=0.90$, (c) $\alpha=1$ which is the exact solution and Plots of $U(x, t)$ or $\mathrm{V}(\mathrm{x}, \mathrm{t})$ versus $t$ at $x=1$ for different values of $(--) \alpha=0.75,(-) \alpha=0.90,(-) \alpha=1$ which is the exact solution. 